\title{
Polarization-Maintaining Hybrid Erbium-Brillouin Amplifier for High-Power Low-Noise Sources
}

\author{
Shane J. Strutz, Member, IEEE, Keith J. Williams, Member, IEEE, and Ronald D. Esman, Fellow, IEEE
}

\begin{abstract}
A low-noise high-power source incorporating a polarization-maintaining erbium-brillouin fiber amplifier is presented. The output noise power is analyzed and a theoretical noise spectral density is determined. Shot-noise-limited performance is predicted and experimentally achieved within the 1-8-GHz-offset frequency range. The polarization extinction ratio was measured as $25 \mathrm{~dB}$.
\end{abstract}

Index Terms-Amplifier noise, brillouin scattering, noise, optical fiber amplifiers, brillouin scattering.

\section{INTRODUCTION}

H IGH-PERFORMANCE photonic links are generally improved by utilizing high photocurrents. Erbium-doped fiber amplifiers (EDFA) [1] may be used to increase the optical power of a fiber link, but the signal-spontaneous beat noise and excess noise from the amplifier degrades the link performance [2]. For externally modulated links, high-power, low relative intensity noise (RIN), solid-state lasers may be used, but are bulky, expensive and limited to a few hundred milliwatts. As an alternative, we have recently developed a hybrid erbium-brillouin (HEB) fiber amplifier, enabling shot-noise-limited performance between $500 \mathrm{MHz}$ and $8 \mathrm{GHz}$ [3]. In this letter, we extend our work from a single-mode amplifier to a polarization-maintaining (PM) version, essential for external modulation. Experimental results for a PM-HEB amplifier that combines the high-output power of an EDFA with the narrow-gain bandwidth of a brillouin amplifier are presented.

\section{SYSTEM CONFIGURATION}

The PM-HEB amplifier is shown schematically in Fig. 1. Light from an inexpensive 30- $\mathrm{mW}$ distributed feedback (polarization extinction $>25 \mathrm{~dB}$ ) is divided into two paths (50/50) with a PM fiber coupler. In one path, the light is amplified by a PM-EDFA, passed through a PM optical circulator (125- $\mu \mathrm{m})$ and sent into one end of a 1-km spool of $80-\mu \mathrm{m}$ elliptical-core PM fiber to pump the brillouin acoustic wave. The 200-mW output from the EDFA is well above the 66-mW stimulated-brillouin-scattering (SBS) threshold for a 1-km spool of $80-\mu \mathrm{m}$ PM fiber at $1556 \mathrm{~nm}$ (Fig. 2). Light following the second path is modulated at the brillouin frequency $(10.363 \mathrm{GHz})$ by a lithium-niobate phase modulator $(V \pi=5.6 \mathrm{~V})$ and used as a

Manuscript received November 1, 2000; revised May 24, 2001. This work was supported by the Office of Naval Research.

The authors are with the Naval Research Laboratory, Code 5652, 4555 Overlook Avenue, SW, Washington, DC 20375 USA (e-mail: strutz@ccs.nrl.navy.mil).

Publisher Item Identifier S 1041-1135(01)07510-3.

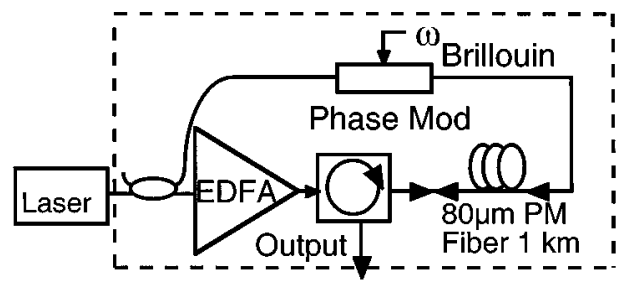

Fig. 1. Experimental configuration. The hybrid amplifier is contained within the dashed box.

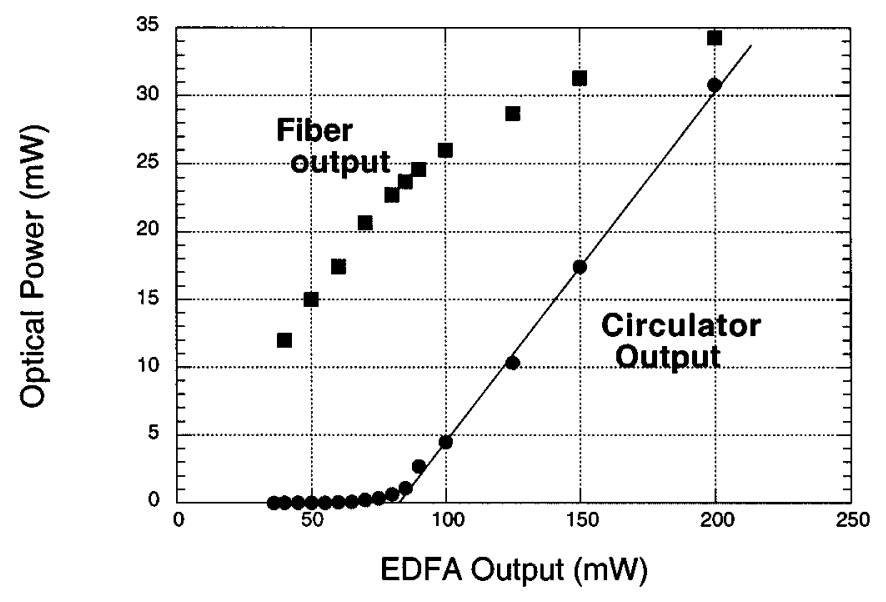

Fig. 2. Brillouin amplifier output powers versus EDFA output power. Pump power to brillouin fiber is $1 \mathrm{~dB}$ less than the EDFA power due to circulator loss. The EDFA output and fiber output were used to determine the brillouin threshold power.

seed input (6-mW) to the brillouin amplifier. The pump beam scatters off of the acoustic grating in the PM fiber, amplifying a $10.363-\mathrm{GHz}$ (seed) sideband of the phase-modulated light. The resulting $32-\mathrm{mW}$ output is limited by both the $200-\mathrm{mW}$ EDFA, the 3-dB optical loss induced by double passing the PM circulator, and the loss $(0.5 \mathrm{~dB})$ from the $125-\mu \mathrm{m}$ (PM circulator) to $80-\mu \mathrm{m}$ fiber (brillouin) splice. Much higher powers are expected with the use of a high-power EDFA, and a single type of PM fiber. Due to the narrow brillouin gain bandwidth $(<35 \mathrm{MHz})$, the added noise from the EDFA is substantially reduced at frequencies greater than $1 \mathrm{GHz}$ from the amplified seed light, thus enabling shot-noise-limited performance.

The noise performance of the PM erbium-brillouin amplifier is determined by comparing three separate sources. The first source consisted of the present PM-HEB source described above (Fig. 1). The second source was amplified by a single PM-EDFA. The third source was a low-noise Nd:YAG laser $(1.319 \mu \mathrm{m}, \mathrm{RIN}<-165 \mathrm{~dB} / \mathrm{Hz})$ which exhibits shot-noise-limited performance above $100 \mathrm{MHz}$ for photocurrents in excess of 
$10 \mathrm{~mA}$. The third source is used as a shot-noise-limited reference throughout the analyses in this letter.

\section{THEORY}

Spontaneous emission from an optical amplifier is converted into electrical noise. This spontaneous emission noise mixes with itself and with signal light at a photodetector (PD); such that, the total output noise ( $\left.N_{\text {Total }}\right)$ of the PD includes: shotnoise $\left(N_{\text {shot }}\right)$, the signal-spontaneous $\left(N_{\text {sig-sp }}\right)$ beat noise, and the spontaneous-spontaneous $\left(N_{\mathrm{sp}-\mathrm{sp}}\right)$ beat noise

$$
N_{\text {Total }}=N_{\text {Shot }}+N_{\text {sig-sp }}+N_{\text {sp-sp }} \text {. }
$$

The $N_{\text {sig-sp }}$ and $N_{\text {sp-sp }}$ of optical amplifiers are given by [4]

$$
N_{\mathrm{sp}-\mathrm{sp}}=2 n_{s p}^{2}(G-1)^{2} e^{2} B_{0}
$$

and

$$
N_{\text {sig-sp }}=\frac{4 e^{2}}{h \nu} P_{\mathrm{in}} n_{\mathrm{sp}}(G-1) G
$$

respectively, where

$$
\begin{array}{ll}
n_{\mathrm{sp}} \sim 500 & \text { spontaneous emission factor; } \\
G & \text { single-pass gain; } \\
e & \text { electronic charge; } \\
\nu & \text { optical wavelength; } \\
h & \text { Plank's constant; } \\
B_{0} & \text { electronic bandwidth. }
\end{array}
$$

The spontaneous emission from the EDFA passes directly through the PM fiber spool since it is too weak to create an acoustic grating; therefore, the primary source of spontaneous noise in our amplifier is the emission from the brillouin amplification segment. Thus, to determine the output noise power of a PM-HEB amplified source, it is necessary to determine the brillouin gain of the PM-HEB amplifier.

A quantitative treatment of the PM-HEB noise properties starts with the SBS equations. The equations describing the propagation of the pump and Stokes waves of a brillouin amplifier relative to the position $z$ along the fiber are [5]

$$
\begin{aligned}
\frac{d I}{d z} & =-g_{B} B I-\alpha_{0} I \\
\frac{d B}{d z} & =-g_{B} B I+\alpha_{0} B
\end{aligned}
$$

where

$$
\begin{array}{ll}
I \text { and } B & \text { pump and Stokes intensities, respectively; } \\
\text { parameter } g_{B} & \text { brillouin gain coefficient; } \\
\alpha_{O} & \text { linear loss. }
\end{array}
$$

From Lichtman [6] and Mao [7] the resulting single-pass gain, $G$, for a pump spectrum $P_{\text {Pump }}(\nu, z)$ is

$$
G\left(\nu_{S}\right)=\int_{0}^{L} P_{\text {Pump }}(\nu, z) \frac{g_{B}}{A_{\text {eff }}} \mathcal{L}\left(\nu-\nu_{S}-\nu_{B}\right) d \nu_{S} d z
$$

where

$$
\mathcal{L}(\nu)=\frac{1}{1+\left(\frac{\nu}{\Delta \nu_{B} / 2}\right)^{2}}
$$

Assuming a narrow-band undepleted pump the single-pass gain becomes

$$
G\left(\nu_{S}, L\right)=\frac{g_{B}}{A_{\mathrm{eff}}} P(0) L_{\mathrm{eff}} \mathcal{L}\left(\nu_{P}-\nu_{S}-\nu_{B}\right)
$$

where $L_{\mathrm{eff}}=\left(1-e^{-\alpha L}\right) / \alpha$, and $A_{\mathrm{eff}}$ is the mode field diameter of the fiber. However, this neglects the effect of pump depletion.

To realize the effects of pump depletion, we continue with the treatment of Bayvel et al. [5]. In their treatment, the formulas in (4) are normalized to the length parameter $\zeta=z / L$, and the optical intensities are normalized to the input pump power ( $P=I / I_{0}$ and $\left.S=B / I_{0}\right)$ resulting in

$$
\begin{aligned}
& \frac{d P}{d \zeta}=-\sigma S P-\beta P \\
& \frac{d S}{d \zeta}=-\sigma S P+\beta S
\end{aligned}
$$

where $\sigma=g_{B} L I_{0}$ is the gain factor and $\beta=\alpha_{0} L$ is the loss.

The optical power of the pump decreases as it propagates through the brillouin medium, causing it to eventually decrease below the brillouin threshold of the fiber. Thus, for simplicity, we divide the single-pass brillouin gain, $G$, into two distinct terms: the nonlinear depleted pump term where the pump power is below $P_{\text {threshold }}$ and the undepleted pump term where the $P>P_{\text {threshold. }}$. The interface between the two regions is determined by setting $P\left(\zeta^{\prime}=z^{\prime} / L\right)=P_{\text {threshold }}$.

We approximate the depleted condition such that $\sigma S \gg \beta$. With strong depletion $S(0)=R P(0)$ so that for positions near $z=0$ (8) become

$$
\frac{d P}{d \zeta}=\frac{d S}{d \zeta}=-\sigma S P=-\sigma R P^{2}
$$

where $R$ represents the percentage of the pump intensity converted into the Stokes wave.

The solution to (9) gives

$$
S(\zeta)=\frac{R}{1+\sigma \zeta}
$$

and

$$
P(\zeta)=\frac{1}{1+\sigma R \zeta} .
$$

Using (10) and (11), the single-pass gain is represented by

$$
\begin{aligned}
G\left(\nu_{S}\right)=\frac{g_{B} \mathcal{L}}{A_{\mathrm{eff}} R}\left\{\int_{0}^{z^{\prime}} I_{\mathrm{in}}\left(1+g_{B} I_{\mathrm{in}} R z\right)^{-1} d z\right. & \\
& \left.+\int_{z^{\prime}}^{L} I\left(z^{\prime}\right) e^{-\alpha z} d z\right\}
\end{aligned}
$$

where the term on the left represents the region of pump depletion and the term on the right represents the region of undepleted pump giving

$$
G\left(\nu_{S}\right)=\frac{g_{B} \mathcal{L}}{R} \frac{\ln \left(1+g_{B} I_{\mathrm{in}} R z^{\prime}\right)}{A_{\mathrm{eff}}}+\frac{g_{B} \mathcal{L} I\left(z^{\prime}\right) L_{\mathrm{eff}}^{\prime}}{A_{\mathrm{eff}}} .
$$

The output noise of the system is found by inserting (13) into (1)-(3). 


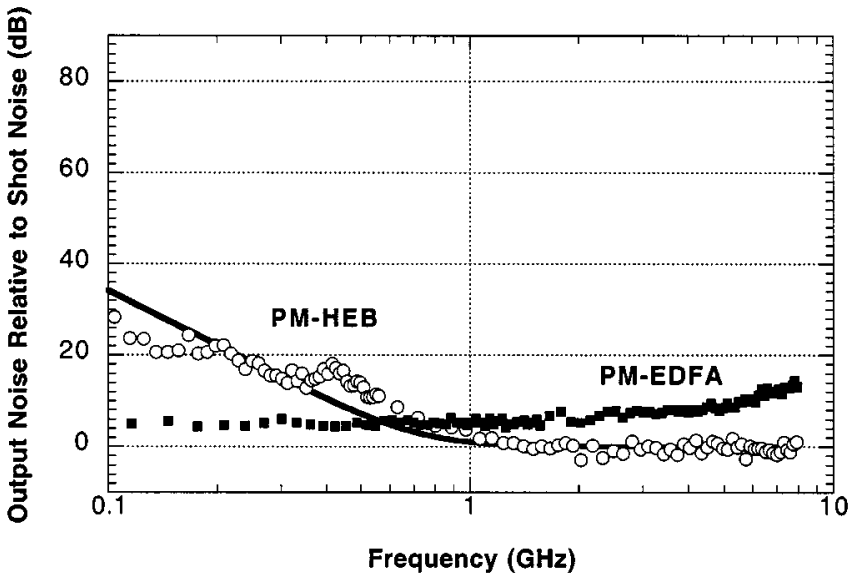

Fig. 3. Output level relative to shot-noise with phase-modulated seed beam. The solid line represents the calculated output noise level. Both sources were measured with $10 \mathrm{~mA}$ of photocurrent.

\section{RESULTS AND DISCUSSION}

We first measured the brillouin threshold of the $80 \mu \mathrm{m}$ PM-fiber $(1 \mathrm{~km})$ without the phase modulator (Fig. 1). As shown optical threshold is reached at $66 \mathrm{~mW}$, with a $\sim 1-\mathrm{dB}$ loss when passing through the PM circulator. For pump powers above $66 \mathrm{~mW}$, the reflected power increases with the input power, and the transmitted intensity decreases. The threshold of our current system is considerably higher than the $<2-\mathrm{mW}$ threshold measured for our previous system, primarily due to the decrease in fiber length (25 to $1 \mathrm{~km})$.

Fig. 3 shows the output-noise power of the hybrid amplifier and the EDFA amplified links relative to the Nd: YAG laser link. It is important to note that the photocurrent for each link was $10 \mathrm{~mA}$ and that the EDFA was seeded with the same light intensity for each link. At a photocurrent of $10 \mathrm{~mA}$, the EDFA amplified link was 5-10 dB above the shot-noise limit; whereas, the link incorporating the hybrid amplifier exhibited shot-noiselimited performance between 1 and $8 \mathrm{GHz}$. The measured results are in close agreement with the calculated output noise level. Much like our non-PM amplifier, this system filters the spontaneous emission from the EDFA and the high-frequency components of the amplified laser RIN since these noise powers are below the SBS threshold. These two effects combine to decrease the detector noise for frequencies greater than $1 \mathrm{GHz}$, a significant improvement over the noise performance of the EDFA-only link. The extinction ratio measured $25 \mathrm{~dB}$.

Though shot-noise-limited performance was achieved, the PM system did not perform as well as the non-PM system which exhibited shot-noise-limited performance down to $500 \mathrm{MHz}$. This appears to be caused by the shorter fiber length used in our PM amplifier. Increasing the fiber length reduces the brillouin threshold, causing the bandwidth of the brillouin amplification to decrease from $20 \mathrm{MHz}$ for $1 \mathrm{~km}$ to as low as $6 \mathrm{MHz}$ for an unseeded 25-km length of single-mode fiber, SMF-28.

We also examined the impact of the phase-modulated signal beam. Fig. 4 shows that injecting a phase-modulated signal

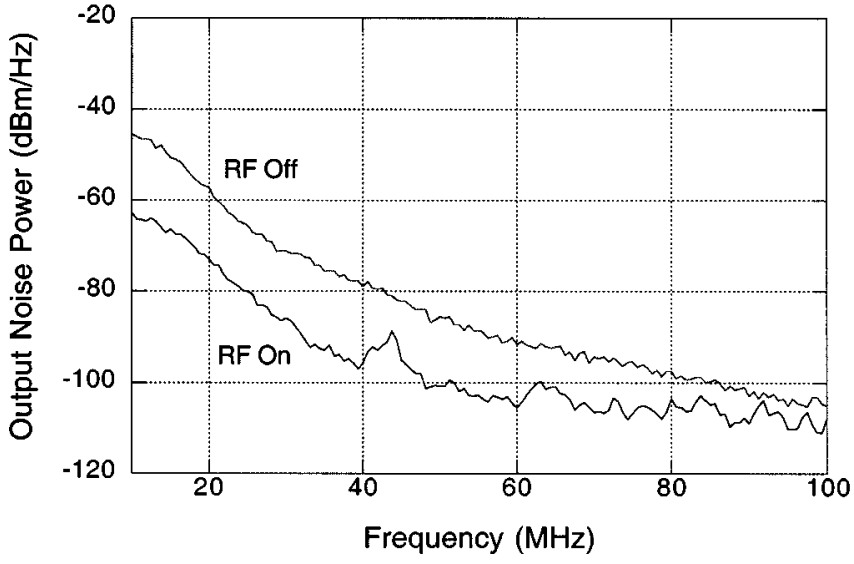

Fig. 4. Noise power with and without phase modulation.

beam (RF On) into the brillouin section of the hybrid amplifier caused an overall reduction in the noise of the amplifier for frequencies below $1 \mathrm{GHz}$. Variations in the output noise of SBS-filtered photonic links have been [3], [4], [8], [9] observed in the past. The seed signal appears to enhance the stability of the acoustic grating in the fiber since the Stokes wave is no longer initiated from noise. This results in a narrower, more stable amplified output signal.

\section{CONCLUSION}

We have improved our HEB amplifier to include PM operation. The simple photonic link amplified with our PM amplifier exhibits shot-noise-limited performance between 1 and $8 \mathrm{GHz}$, and a polarization extinction ratio of $25 \mathrm{~dB}$. Our amplifier enables high-power low-noise photonic links without the need for expensive solid-state lasers. Though not demonstrated here, this amplifier is predicted to provide much higher output powers if a high-power EDFA is utilized.

\section{REFERENCES}

[1] R. W. Tkach and A. R. Chraplyvy, "Fiber brillouin amplifiers," Opt. Quantum. Electron., vol. 21, pp. 105-112, 1989.

[2] N. A. Olsson and J. P. Van Der Ziel, "Fiber brillouin amplifier with electronically controlled bandwidth," Electron. Lett., vol. 22, pp. 488-489, 1986.

[3] S. J. Strutz and K. J. Williams, "Low-noise hybrid erbium-brillouin amplifier," Electron. Lett., vol. 36, pp. 1359-1360, 2000.

[4] P. C. Becker, N. A. Olsson, and J. R. Simpson, Erbium-Doped Fiber Amplifiers. San Diego, CA: Academic, 1999.

[5] P. Bayvel and P. M. Radmore, "Solutions of the SBS equations in single-mode optical fibers and implications for fiber transmission systems," Electron. Lett., vol. 26, pp. 434-436, 1990.

[6] E. Lichtman, R. G. Waarts, and A. A. Friesem, "Stimulated brillouin scattering excited by a modulated pump wave in single-mode fibers," $J$. Lightwave Technol., vol. 7, pp. 171-173, Jan. 1989.

[7] X. P. Mao, R. W. Tkach, A. R. Chraplyvy, R. M. Jopson, and R. M. Derosier, "Stimulated brillouin threshold dependence on fiber type and uniformity," IEEE Photon. Technol. Lett., vol. 4, pp. 66-69, Jan. 1992.

[8] K. J. Williams and R. D. Esman, "Stimulated brillouin scattering for improvement of microwave fiber-optic link efficiency," Electron. Lett., vol. 30, pp. 1965-1966, 1994.

[9] A. L. Gaeta and R. W. Boyd, "Stochastic dynamics of stimulated brillouin scattering in an optical fober," Phys. Rev. A, vol. 44, pp. 3205-3209, 1991. 